\title{
ANALYSIS OF QUEUING APPLICATIONS PERFORMANCE USING MATLAB
}

\author{
Debnath Bhattacharyya $^{1}$, N. Thirupathi Rao ${ }^{1}$, Pilla Srinivas ${ }^{2}$ and Jason Levy ${ }^{3}$ \\ ${ }^{l}$ Department of Computer Science and Engineering, \\ Vignan's Institute of Information Technology, \\ Visakhapatnam-530049, India \\ ${ }^{2}$ Department of Computer Science \& Engineering \\ Dadi Institute of Engineering \& Technology \\ Anakapalle, Visakhapatnam, India \\ ${ }^{3}$ University of Hawaii, \\ Hawaii, USA \\ debnathb@gmail.com,nakkathiru@gmail.com, srinivasp3@gmail.com, \\ jlevy@hawaii.edu
}

\begin{abstract}
Cloud computing is the technology that was gaining the attention of the most of the companies in market and utilization also increasing day to day by almost from companies to the common people. The working of these cloud models is very simple. A huge number of servers are used to store the data and a vast amount of data and the service of providing data to the customers staying at remote locations too. Almost all cloud based models are not free and users need to pay reasonable amount to use the services of these clouds. As the huge data is stored in these servers and the usage of this data by a huge number of customers, there is a chance of overcrowded at servers. Important data or the hot data like the new movies, exam results or bank transactions, etc. can have the most of the crowds at various time intervals. Hence, it is required to analyze the number of customers are being using the current cloud models at different intervals of time. Based on the results, the adjustments or the changes in the network model can be completed. In the current article, an attempt has been made to analyze a cloud model by considering the model working in study state and the performance was analyzed for two queuing models. Several queuing models are available in research to analyze the performance of a queuing model. In the current article, the queuing models considered are $M / M / 1$ and $\mathrm{M} / \mathrm{M} / \mathrm{c}$ models. The performance of the queuing models are analyzed with various performance metrics of a network or the cloud model are arrival rates to the model, service rates to the model, traffic density, throughput etc. The results are displayed in the results section.
\end{abstract}

Keywords - Cloud computing, queuing models, exponential distribution, M/M/1, $\mathbf{M} / \mathbf{M} / \mathbf{c}$

\section{INTRODUCTION}

Cloud computing is one of the interests gaining and mostly used technologies in market today. The storage space is becoming costly day to day, most of the companies or public are looking towards the cloud centers such that to store their vast data. A cloud center may have a huge number of servers with huge capacity to store data [1]. The

Received: March 5, 2019

Reviewed: May 29, 2019

Accepted: June 2, 2019

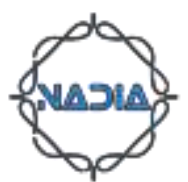


facility was open to all types of customers. It is very easy to store the data and also to retrieve the data from a cloud center. Almost all big companies in the market are maintaining their own data storage centers. Nowadays, the mobile phone companies like Redmi and other companies also using these cloud centers such that to give their customers to provide extra space such that to store their data more than the capacity of those mobile phones[2]. The mobile companies like the Redmi, Mi are providing such facilities to their customers. The users of these companies mobile phones can use the space allocated to them by simply login to their account and can use their space for the lifetime. It's a marketing strategy to attract the customers too. In general, the cloud service providers will charge some specific charges to store and use the space of their servers. The users who have the proper login details can use the service. The other advantage of choosing these cloud models are the damage to data due to various problems. The data stored in cloud centers cannot be damaged due to the storage of data will be in various servers at various locations. If any issue or any damage occurs to any server or cloud, the data can be retrieved from any other server that was connected in the same cloud [3,6,7].

In recent days, the utility of the internet and its related applications had increased a lot. As the usage is increased, almost all the applications are available in the internet. As a result, the space for storage of such applications is growing day by day. As a result, the more number of servers to be established and to be provided by service providers. The cost of establishing such large type of servers, space and maintenance of such servers is also growing day by day. As a result of these factors, the cloud utility was not made free. Now a day, the number of customers' using these cloud centers had increased a lot. Both the private organizations and government organizations also using these cloud centers. Especially from the government pointy of view, the maintenance is the major problem for any organization and government services are almost providing the services from various cloud centers. As a result, the network traffic to these clouds is increasing a lot during some intervals of the time period. Now a day, movies are being stored in cloud centers for more security. The government schemes, student's results and various govt. beneficiary schemes also being stored in these cloud centers. The traffic to such cloud centers are more during the peak hours and normal at other hours of time. The customers entering into the cloud model and coming out from the cloud models can be symbolized as a queue line model. As a result, in the current article, the arrival of data packets to the network model are assumed to be following queuing models and tried to analyze the performance of the model $[4,5]$.

\subsection{CLOUD DATA CENTERS}

The storing and accessing of data of a company is more cost effective than setting up a new cloud center for any organization or a company. Hence, always a thought was given on getting the service from cloud center is easier than establishing a center and maintain such center for any individual company. This cloud center is a physical center with a number of servers located at different locations to store the data [6]. The maintenance and the other services required to maintain such centers will be taken care by the cloud center people itself. Those companies have no role or no expense on such issues. These are some of the main reasons that most of the companies are looking towards cloud centers utilization rather than establishing o own cloud center. The other benefits that they are getting by using the sources of the cloud center are the security that was provided to these cloud servers. In normal cases, the companies need to expense a lot on providing security to these servers, but here the security of these centers will be taken care by the cloud center people [7]. As a result, the less expense on security to the center and the security of the cloud center will be taken care by the loud center people itself. The data stored in a cloud center may not belong to only one company, several other companies also storing their data. It is the responsibility of the cloud center people to provide security. As a 
result, the customers can happily store their data and can use the services from the cloud center [8].

\subsection{QUEUING SYSTEMS}

In general, people used to stand in various queues to buy or purchase something or in some other cases to pay or get some items. The queue lines are formed such that to provide the service one by one such that everyone in the queue can get the service. Also the service can be provided neat with more clear and clarity on the type of service being provided. The queue lines follow two types of schemes like LIFO and FIFO [7, 9]. The first in first out and last in first out is available for us to implement. The second model is used in almost all the services being provided worldwide for a queuing model. Wherever, the lines are developed or proposed to provide the service, the queuing models can be considered [11, 12, 13]. In the current work also, the packets being transferring from one node to the other node are in the form of a queue line. Similarly, the customers who were approaching this cloud center are also in the form of a queue and the service by the center will be given in the same order of arrival of requests from the customers to the center. The data that was given as input to be considered in the form of a queuing model input. Several queuing models are available in literature such that to study the performance of the model. Several authors had considered several models such that to identify the performance of the queuing models $[10,14]$. In the similar, in the current article, two models are considered such that the input to the current cloud model and those are M/M/1 and $\mathrm{M} / \mathrm{M} / \mathrm{c}$ models $[6,8]$. With the help of various performances measuring metrics, an attempt has been made to analyze the performance of the cloud model with various set of inputs. The queuing model can be observed as follows,

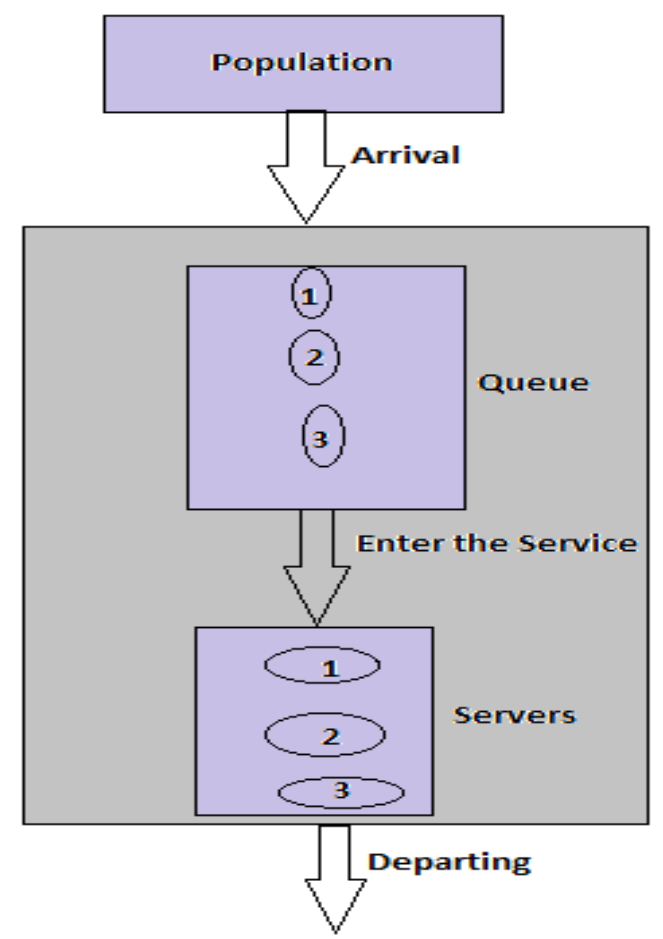

Fig. 1 Queuing System

In order to fit the queuing models to the network models, several authors had considered several assumptions. The most important consideration was the flow of packets to the network or the server is flowing in the form of first in first out. The packets 
which were arriving first for the service, these packets or the data need to be processed first and the other packets later in the form of the queue. All these queuing models are independent, they follow the independent distributions and exponential distribution in some other cases.

\section{LITERATURE REVIEW}

Some of the authors and their contributions towards the current work are discussed as follows,

Shaguna Gupta and Shakshi Arora (2018) discussed in detail about the queuing models and their utilization in cloud computing models and applications. They discussed about the importance of the cloud computing applications and their performance and their utilization in various sections of the research. They had also discussed about the Quality of Service and how the researchers can provide the best quality of service to the customers whoever is using these cloud services.

K.Ruth Evangelin and V.Vidhya (2015) discussed about the various performance metrics to be considered for evaluating the performance of any cloud computing model or application. They had discussed in detail about both cloud computing user and cloud computing service provider. They had analyzed the performance of the model with $\mathrm{M} / \mathrm{M} / \mathrm{C}$ model for single servers.

Jordi Vilaplana, Francesc Solsona (2014) discussed about the provision of providing the good quality of service to the customers by using various cloud models. These cloud models performance and working can be better understood by the utilization of queuing models. Here, the cloud model platforms are developed on the basis of open Jackson network which can be used for wireless networks. Based on the results obtained for the performance metrics of such networks, the performance of the networks can be understood better.

Lizheng Guo, Tao Yan, Shuguang Zhao and Changyuan Jiang (2014) had discussed about the performance of the cloud models with queuing based model with the help of optimization. They had utilized the optimized model for the cloud applications such that the network performance can be understood easily. Several performance metrics had been used for analyzing the performance of the considered model. The optimized performance of the model was been obtained for the queuing model $\mathrm{M} / \mathrm{M} / \mathrm{m}$.

\section{PROBLEM DESCRIPTION AND SOLUTION}

From literature review, several authors had considered the usage of queuing models for analyzing the performance of cloud computing based applications and models. Some authors had used the $\mathrm{M} / \mathrm{M} / \mathrm{m}$ models with multi server model and some other authors had used M/MC model with single server model. Hence, from the existing works observations, in the current article, an attempt has been made to model the cloud computing network models with $\mathrm{M} / \mathrm{M} / 1$ and $\mathrm{M} / \mathrm{M} / \mathrm{c}$ queuing models for single server models with study state analysis. In the previous works, not much authors discussed about the time dependant performance of the models. But, in practical applications, the performance of the model with respect to time is more important. Hence, in the current article an attempt has been made to consider the performance of the both models with respect to time also needs to be analyzed. Hence, several performance metrics like throughput, delay in packets, number of customers in queues, number of packets in queue etc. are used to verify such that the performance of the model can be understood better for further details. 


\section{SYSTEM DESIGN}

System design includes parameters, performance measures, stability and properties considered for the data center performance evaluation using queuing models. The detailed working flow of the model considered is as follows,

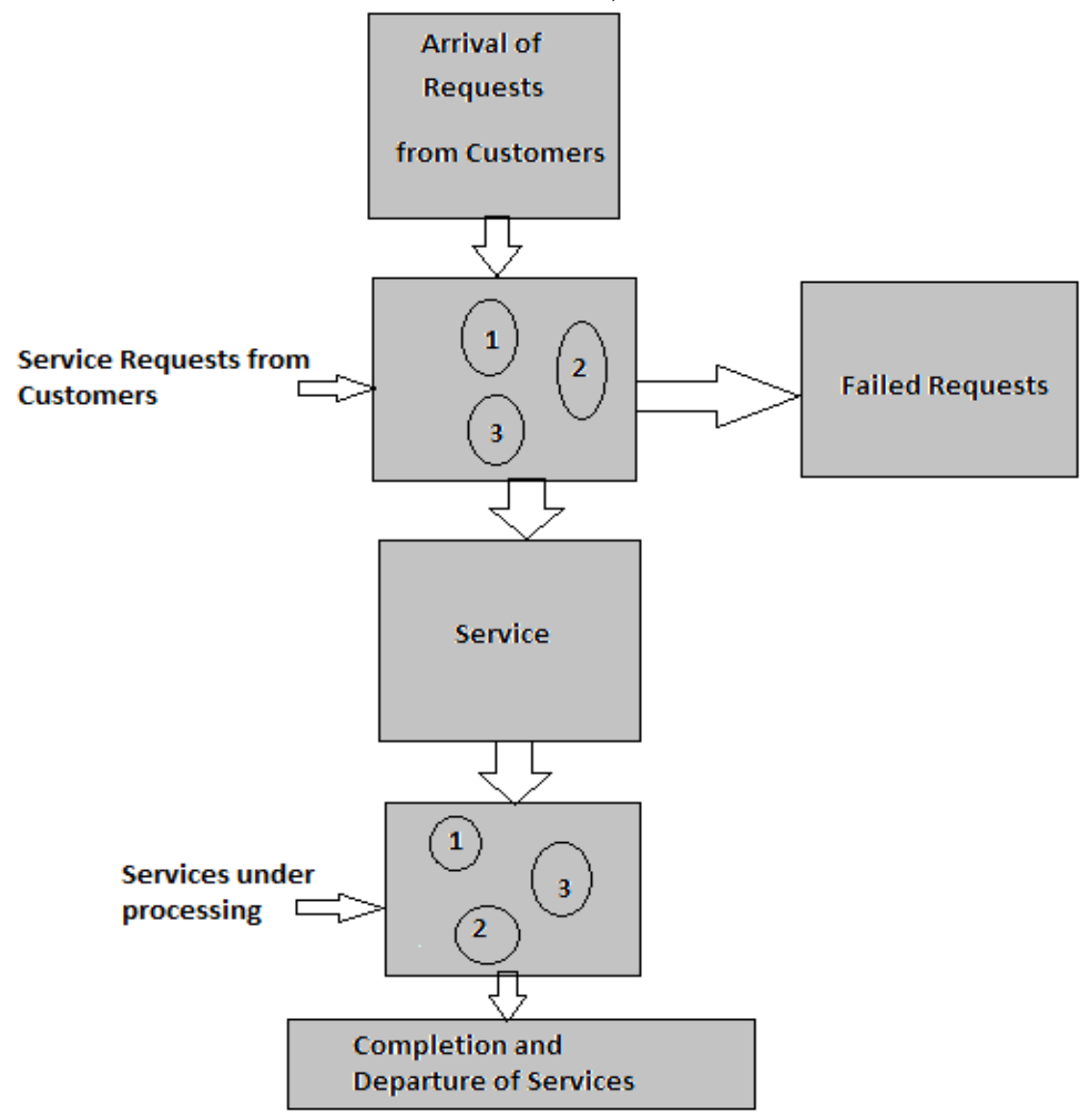

Fig 2 Flow Diagram of the Considered Cloud Model

System parameters are,

a. It is customary to introduce some notation for the performance measures of interest in queuing systems.

b. The Number of customers in the system: $\mathrm{LS}_{\mathrm{S}}$

c. Number of customers in the queue: $\mathrm{L}_{\mathrm{Q}}$

d. Response Time: $\mathrm{W}_{\mathrm{S}}$

e. Waiting Time $\mathrm{W}_{\mathrm{Q}}$

To evaluate the performance parameters of the data center in cloud architecture, programs consider the following input values:
i. Interarrival rates
ii. Service rates
iii. The Number of servers, $c$
iv. Maximum number of customers allowed, $K$

The list of output parameters of the programs are highlighted below:

i. Length of customers in a system, $L_{S}$

ii. Length of customers in queue, $L_{Q}$

iii. Waiting time of customers in a system, $W_{S}$ 


\section{IMPLEMENTATION}

If any system working with no clarity or no good opinion on the arrival of data to a networking system or communication system, or if any doubts or no clarity on the performance of the system or communication systems about the service times, then the good option for researchers to work for the queuing models. By using the queuing models with the combination of various other distribution patterns, the performance of the communication networks or the machines can be understood and realized very soon. Various services will be provided by the communication networks, in order to achieve these performances, several performance metrics are considered. In the current work, a cloud computing model based data center is considered and the arrival of packets are discussed in detail and tried to analyze the performance of such cloud model data center. The MATLAB software used to analyze the performance.

\subsection{QUEUING MODELS}

\section{A) QUEUING MODEL - M/M/1}

The queuing model considered in the first model is the $\mathrm{M} / \mathrm{M} / 1$ model. It has Interarrival rates. This Interarrival also has exponentially distributed relation with the Erlang distribution.

Steady state performance measures for the above model are:

$$
\begin{aligned}
& L_{S}=\rho+\frac{\left(1+\frac{1}{e r}\right) \rho^{2}}{2(1-\rho)} ; \quad L_{O}=\frac{\left(1+\frac{1}{e r}\right) \rho^{2}}{2(1-\rho)} \\
& W_{S}=\frac{1}{\mu}+\frac{\left(1+\frac{1}{e r}\right) \rho}{2 \mu(1-\rho)} ; \quad W_{Q}=\frac{\left(1+\frac{1}{e r}\right) \rho}{2 \mu(1-\rho)} \\
& \operatorname{Var}\left(L_{s}\right)=\frac{\rho^{2}(1+e r)(2+e r)}{3 e r^{2}(1-\rho)}+\frac{\rho^{2}\left(1+\frac{1}{e r}\right)}{2(1-\rho)}+\rho^{2}(3-2 \rho) \frac{\left(1+\frac{1}{e r}\right)}{2(1-\rho)}+\rho(1-\rho) \\
& \operatorname{Var}(W s)=2 W q^{2}+\frac{\rho(1+e r)(2+e r)}{\mu^{2} 3 e r^{2}(1-\rho)}+\frac{\left(1+\frac{1}{e r}\right)}{\mu^{2}(1-\rho)}-W s^{2} \\
& \operatorname{Var}(L q)=\frac{\rho^{2}(1+e r)}{2 e r(1-\rho)}\left(1+\frac{\rho^{2}}{2 e r(1-\rho)}\right)+\frac{2 \rho(2+e r)}{3 e r} \\
& \operatorname{Var}(W q)=W^{2}+\frac{\rho(1+e r)(2+e r)}{\mu^{2} 3 e r^{2}(1-\rho)} \\
& \text { Note: } r(o r) \text { \&r is the notation for Erlang parameter }
\end{aligned}
$$

\section{B) QUEUING MODEL - M/M/C}

The second model considered here in the current work was the M/M/c model. It also has exponential distribution and it has Interarrival rates of transfers and arrivals. The performance of the current model is analyzed with the help of various parameters and discussed as below,

Steady state performance measures for the above model are: 


$$
\begin{aligned}
& B(c, \rho)=\frac{\frac{\rho^{c}}{c !}}{1+\frac{\rho^{2}}{2 !}+\frac{\rho^{3}}{3 !} \ldots+\frac{\rho^{c}}{c !}} \quad \text { (Erlang B formula) } \\
& L_{S}=\frac{\lambda(1-B(c, \rho)}{\mu} ; \quad L_{Q}=0 \\
& W_{S}=\frac{1}{\mu} ; \quad W_{O}=0
\end{aligned}
$$

\section{D). QtsPlus4Calc Software (http://qtsplus4calc.sourceforge.net):}

In order to analyze the performance of the current model, the software entitled QtsPlus4Calc was used and the home page model of the software is shown as follows,

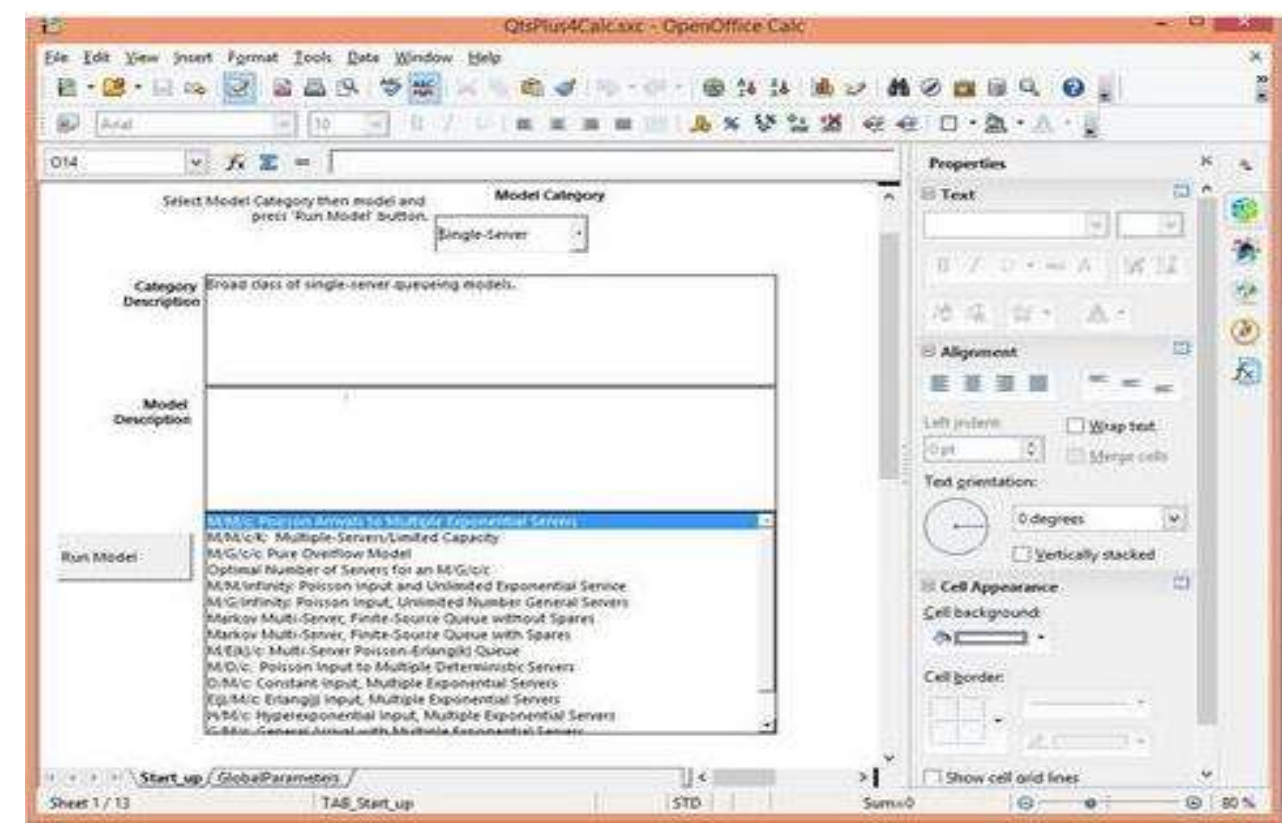

Fig. 2 QtsPlus4Calc Environment Screenshot

\section{RESULTS AND DISCUSSION}

In order to analyze the performance of the current two queuing models with cloud based model, several input parameters are considered and the performance in the name of various factors was analyzed and tabulated. The detailed performance can be observed from various graphical representations at below. The below tables and graphical representations are as follows,

Table I. Performance of data center $-\mathrm{M} / \mathrm{M} / 1$, model $=1$

\begin{tabular}{|c|c|c|c|c|c|c|c|c|c|c|c|}
\hline \multicolumn{10}{|c|}{ Performance of data center - M/M/1 model=1, Er =6 } \\
\hline \multirow{2}{*}{$\mathrm{t}$} & & & & \multicolumn{7}{c|}{ Input Data } & \multicolumn{5}{c|}{ Variance } \\
\cline { 2 - 14 } & $\boldsymbol{\Lambda}$ & $\boldsymbol{\rho}$ & $\begin{array}{c}\text { Er- } \\
\text { parameter }\end{array}$ & $L s$ & $L q$ & $W s$ & $W q$ & $\operatorname{Var}(L s)$ & $\operatorname{Var}(W s)$ & $\operatorname{Var}(L q)$ & $\operatorname{Var}(W q)$ \\
\hline 1 & 0 & 0 & 6 & 0.00 & 0.00 & 1.00 & 0.00 & 0.00 & 0.50 & 0.00 & 0.00 \\
\hline 1 & 0.1 & 0.1 & 6 & 0.11 & 0.01 & 1.08 & 0.08 & 0.11 & 0.62 & 0.01 & 0.12 \\
\hline 1 & 0.2 & 0.2 & 6 & 0.24 & 0.04 & 1.19 & 0.19 & 0.27 & 0.79 & 0.08 & 0.29 \\
\hline 1 & 0.3 & 0.3 & 6 & 0.40 & 0.10 & 1.32 & 0.32 & 0.49 & 1.03 & 0.26 & 0.53 \\
\hline
\end{tabular}




\begin{tabular}{|c|c|c|c|c|c|c|c|c|c|c|c|}
\hline 1 & 0.4 & 0.4 & 6 & 0.60 & 0.20 & 1.50 & 0.50 & 0.83 & 1.42 & 0.67 & 0.92 \\
\hline 1 & 0.5 & 0.5 & 6 & 0.88 & 0.38 & 1.75 & 0.75 & 1.39 & 2.06 & 1.52 & 1.56 \\
\hline 1 & 0.6 & 0.6 & 6 & 1.28 & 0.68 & 2.13 & 1.13 & 2.45 & 3.27 & 3.29 & 2.77 \\
\hline 1 & 0.7 & 0.7 & 6 & 1.93 & 1.23 & 2.75 & 1.75 & 4.81 & 5.90 & 7.30 & 5.40 \\
\hline 1 & 0.8 & 0.8 & 6 & 3.20 & 2.40 & 4.00 & 3.00 & 11.84 & 13.50 & 18.40 & 13.00 \\
\hline 1 & 0.9 & 0.9 & 6 & 6.98 & 6.08 & 7.75 & 6.75 & 51.58 & 55.06 & 72.14 & 54.56 \\
\hline 1 & 1 & 1 & 6 & Inf & Inf & Inf & Inf & Inf & NaN & Inf & NaN \\
\hline
\end{tabular}

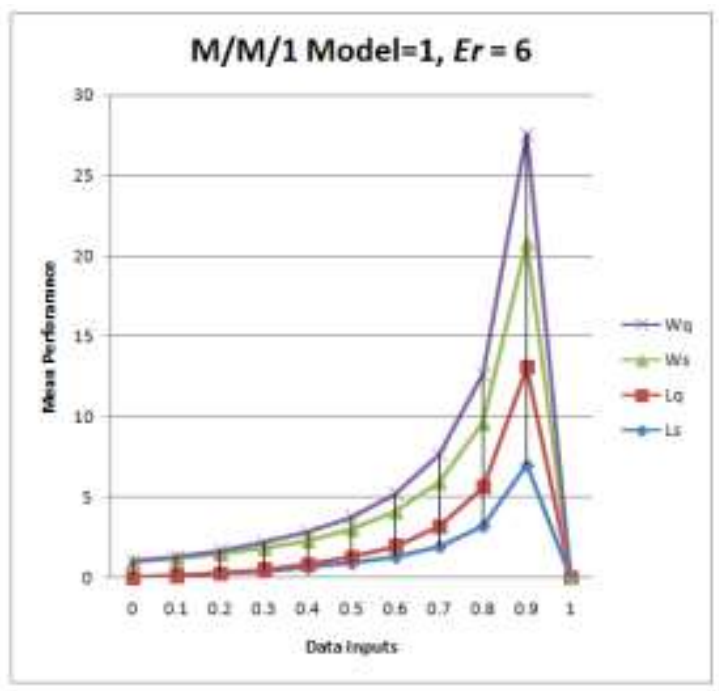

Fig. 3 Graphical Representation of First Model for Mean Performance Measures

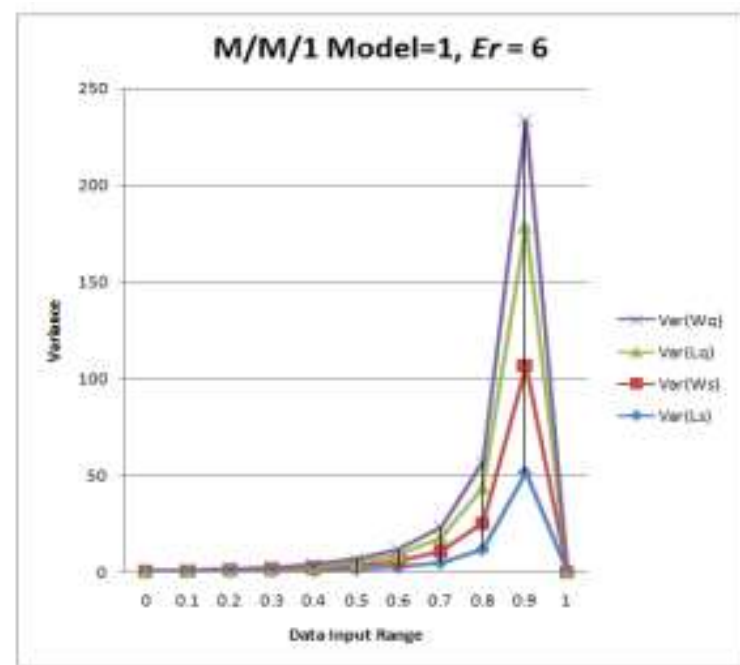

Fig.4 Graphical Representation of First Model for Variance Performance Measures

Table II. Performance of data center $-\mathrm{M} / \mathrm{M} / \mathrm{c}$ model $=2, E r=4$

\begin{tabular}{|c|c|c|c|c|c|c|c|c|c|c|c|}
\hline \multirow{2}{*}{$\mathrm{t}$} & \multicolumn{7}{|c|}{ Inputs } & \multicolumn{3}{|c|}{ Variance } \\
\cline { 2 - 13 } & $\boldsymbol{\Lambda}$ & $\boldsymbol{\rho}$ & $\begin{array}{c}\text { Er- } \\
\text { parameter }\end{array}$ & $L s$ & $L q$ & $W s$ & $W q$ & $\operatorname{Var}(L s)$ & $\operatorname{Var}(W s)$ & $\operatorname{Var}(L q)$ & $\operatorname{Var}(W q)$ \\
\hline 2 & 0 & 0 & 4 & 0.00 & 0.00 & 0.50 & 0.00 & 0.00 & 0.06 & 0.00 & 0.00 \\
\hline 2 & 0.2 & 0.1 & 4 & 0.11 & 0.01 & 0.53 & 0.03 & 0.11 & 0.08 & 0.02 & 0.02 \\
\hline
\end{tabular}




\begin{tabular}{|c|c|c|c|c|c|c|c|c|c|c|c|}
\hline 2 & 0.4 & 0.2 & 4 & 0.23 & 0.03 & 0.58 & 0.08 & 0.25 & 0.11 & 0.13 & 0.05 \\
\hline 2 & 0.6 & 0.3 & 4 & 0.38 & 0.08 & 0.63 & 0.13 & 0.43 & 0.15 & 0.47 & 0.08 \\
\hline 2 & 0.8 & 0.4 & 4 & 0.57 & 0.17 & 0.71 & 0.21 & 0.70 & 0.21 & 1.26 & 0.15 \\
\hline 2 & 1 & 0.5 & 4 & 0.81 & 0.31 & 0.81 & 0.31 & 1.13 & 0.32 & 2.91 & 0.25 \\
\hline 2 & 1.2 & 0.6 & 4 & 1.16 & 0.56 & 0.97 & 0.47 & 1.91 & 0.52 & 6.28 & 0.45 \\
\hline 2 & 1.4 & 0.7 & 4 & 1.72 & 1.02 & 1.23 & 0.73 & 3.60 & 0.96 & 13.50 & 0.90 \\
\hline 2 & 1.6 & 0.8 & 4 & 2.80 & 2.00 & 1.75 & 1.25 & 8.56 & 2.25 & 31.60 & 2.19 \\
\hline 2 & 1.8 & 0.9 & 4 & 5.96 & 5.06 & 3.31 & 2.81 & 36.35 & 9.38 & 103.59 & 9.32 \\
\hline 2 & 2 & 1 & 4 & Inf & Inf & Inf & Inf & Inf & NaN & Inf & NaN \\
\hline
\end{tabular}

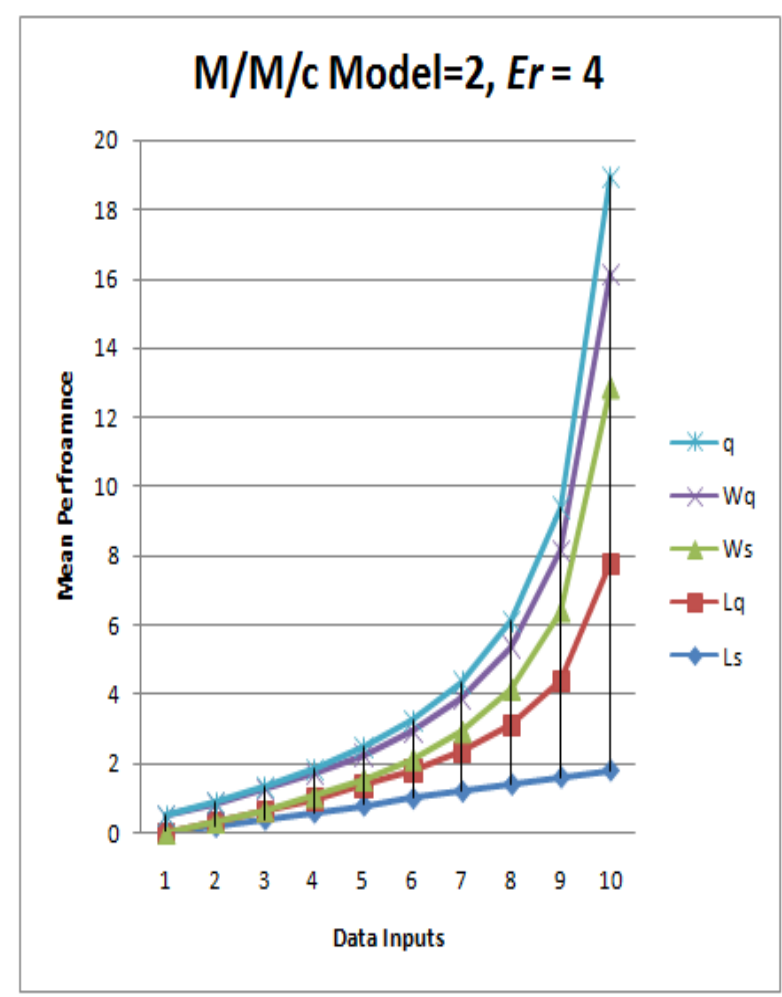

Fig. 5 Graphical representation of Second Model M/M/c for Mean Performance Measures 


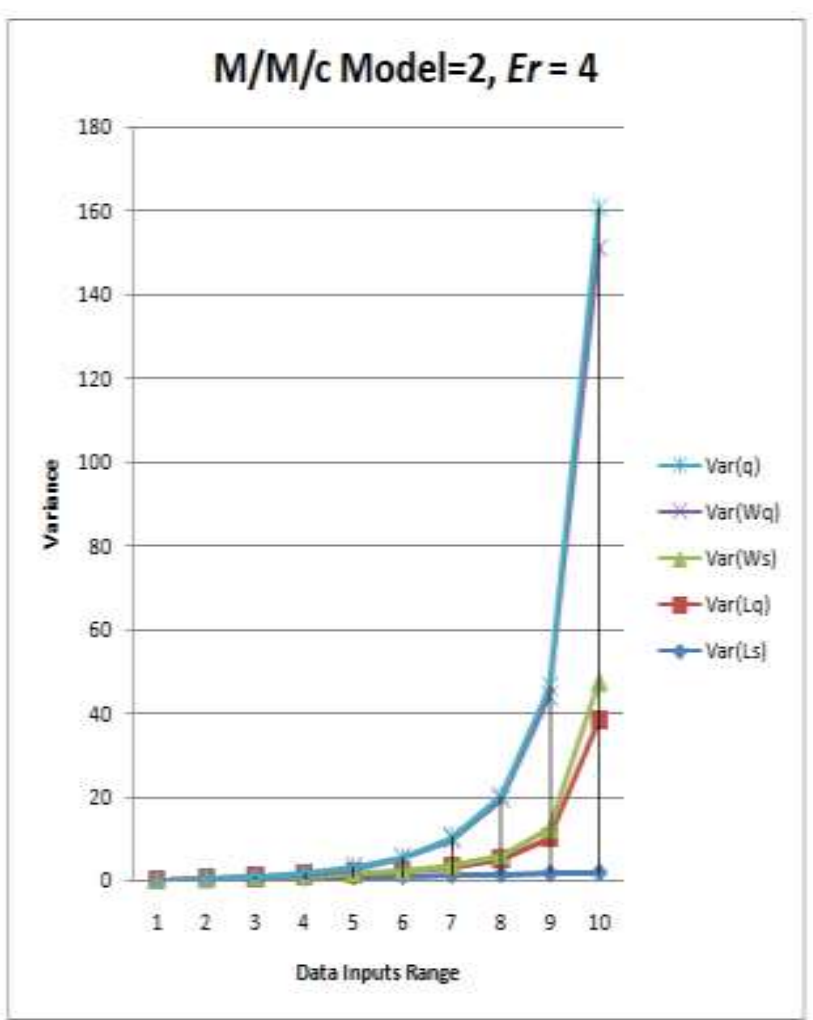

Fig. 6 Graphical Representation of Second Model M/M/c for Variance Performance Measures

The performance of the both models considered here is analyzed by applying various set of inputs. The time considered here is study that is the time period to be considered for the execution of the model or the time taken for the model to be complete given tasks can be taken as study. There is no change in time value until the completion of the considered process. The performance of the both models is impressive. The performance can be observed in tabular formats and for the better understanding the results are shown under graphical representations also.

\section{CONCLUSIONS}

In the current work, an attempt has been made to analyze the performance of cloud model data center with the help of queuing models. Two queuing models have been considered here with study state. In the study state, the arrivals of the packets are following study that is the time for the entire process is fixed and no change later on until the completion of the process. Several parameters have been studied and the performance was given in tabular and graphic al formats. From the results, it is observed that the performance of the models is working efficiently and the results are analyzed in detail.

\section{REFERENCES}

[1] Shaguna Gupta and Shakshi Arora, "Queuing Systems in Cloud Services Management: A Survey", International Journal of Pure and Applied Mathematics, Vol.119, No. 12, 2018, Pp.12741-12753.

[2] K. Ruth Evangelin and V. Vidhya, "Performance Measures of Queuing Models Using Cloud Computing", Asian Journal of Engineering and Applied Technology, Vol. 4, No. 1, 2015, Pp.8-11.

[3] Jordi Vilaplana, Francesc Solsona, "A queuing theory model for cloud computing”, The Journal of Supercomputing, Springer, Vol.69, No.1, 2014, Pp.492-507.

[4] Lizheng Guo, Tao Yan, Shuguang Zhao and Changyuan Jiang, "Dynamic Performance Optimization for Cloud Computing Using M/M/m Queuing System", Journal of Applied Mathematics, 2014, Vol.2014, Pp.1-8. 
[5] Hamzeh Khazaei, Jelena and Vojislav, "Performance Analysis of Cloud Computing Centers Using $\mathrm{M} / \mathrm{G} / \mathrm{m} / \mathrm{m}+\mathrm{r}$ Queuing Systems", IEEE Transactions on parallel and distributed systems, Vol. 23, May 2012.

[6] Hamzeh Khazaei, "Performance Modeling of Cloud Computing Centers", Doctoral dissertation, The University of Manitoba, Canada, Oct 2012.

[7] B. Yang, F. Tan, Y. Dai, and S. Guo., "Performance evaluation of cloud service considering fault recovery", First International Conference on Cloud Computing (CloudCom) 2009, Dec. 2009.

[8] A. Chandrika Sai Priya, "Integrated Framework for Multi-User Encrypted Query Operations on Cloud Database Services", International Journal of Cloud-Computing and Super-Computing, 2016, Vol.3, No.2, GVPress. pp:1-6.http://dx.doi.org/10.21742/IJCS.2016.3.2.01.

[9] Kondapalli Kanaka Rao, "Cloud Computing To Develop Applications”, International Journal of CloudComputing and Super-Computing, Vol.4, No. 1. Jun. 2017.GVPress. pp:914.http://dx.doi.org/10.21742/IJCS.2017.4.1.02.

[10] S Rithika, "Issues on Developing Interoperable Cloud Applications", International Journal of Private Cloud Computing Environment and Management. Vol. 4. No. 1. Apr. 2017.GVPress. pp:914.http://dx.doi.org/10.21742/IJPCCEM.2017.4.1.02.

[11] T. Ravi Kumar, "Advanced Approach and Management in Cloud Trust" International Journal of Urban Design for Ubiquitous Computing. Vol. 4. No. 2. Sep. 2016.GVPress. pp:712.http://dx.doi.org/10.21742/IJUDUC.2016.4.2.02.

[12] P.Harika, N.Thirupathi Rao, "A Survey on Cloud Computing - Investigative Points, Confronts, Design and Applications", International Journal of Cloud-Computing and Super-Computing. Vol. 5. No. 1. Jun. 2018.GVPress. pp:9-16.http://dx.doi.org/10.21742/IJCS.2018.5.1.02.

[13] T. Hari Krishna, "Role of Kernel in Operating System Survey", International Journal of Private Cloud Computing Environment and Management. Vol. 3. No. 1. Apr. 2016.GVPress. pp:1720.http://dx.doi.org/10.21742/IJPCCEM.2016.3.1.03.

[14] Ch L N Deepika, "Data Access Control for Multiauthority Storage System", International Journal of Private Cloud Computing Environment and Management. Vol. 4. No. 1. Apr. 2017.GVPress. pp:18.http://dx.doi.org/10.21742/IJPCCEM.2017.4.1.01. 
International Journal of Advanced Science and Technology Vol.127 (2019) 